\title{
Die Entwicklungsgeschichte der Stoppuhr und ihre Verwendung als Rationalisierungsinstrument
}

\author{
von Sonja Apel
}

\section{Überblick}

In der wissenschaftlichen Forschung über die Rationalisierungsbewegung der 1920er und 1930er Jahre werden die hierfür verwendeten und benötigten Messinstrumente wie etwa Stoppuhren als gegeben betrachtet. Sie werden in keinen eigenen historischen Kontext gesetzt. Auch im Bereich der Uhrengeschichte wurde die Stoppuhr oder der Kurzzeitmesser als Einzelartefakt noch keiner näheren Erforschung unterzogen, obwohl die Stoppuhr, wie die Uhr an sich, das Leben der Menschen nachhaltig verändert hat. Dies manifestiert sich gerade in der Veränderung des Arbeitsprozesses, in dem die Vorkalkulation, die Entlohnung und das Arbeitspensum auf der Auswertung von „gestoppten" Zeiten basieren. Ausgehend vom technischen Objekt und seiner Verwendungsgeschichte im sportlichen, wissenschaftlichen und militärischen Bereich werden Vorläufer, Voraussetzungen und Grundlagen für die in der Rationalisierungsbewegung eingeführte Zeitstudie untersucht.

\begin{abstract}
Among those who study the rationalization movement of the 1920s and 1930s, the stopwatches that were used in time-and-motion studies are often taken for granted. Curiously, the same is true among those who study the historical evolution of timepieces, for even they have yet to subject the stopwatch or the microchronometer to detailed investigation. Like the clock, however, the stopwatch is a tool that has profoundly altered our lives. This is especially true on the shop floor, where preliminary process calculations, payment schedules, and worker tasks are now based on timed sequences. In order to better understand and properly contextualize the tools of the rationalization movement, this paper examines the history of the technology of the stopwatch as well that of as its many uses, from sports to the sciences and from the military to the time-and-motion study.
\end{abstract}

Im Rahmen der Rationalisierungsgeschichte stellt das technische Artefakt Stoppuhr ein oft zitiertes und meist negativ belegtes Symbol hinsichtlich der Veränderungen von Arbeitszeit, Lebenszeit und Disziplin dar. Gerade in Verbindung mit Rationalisierung und Massenproduktion standen die Zeitanalysen 
im Mittelpunkt, „für die die Stoppuhr zum verhassten Symbol wurde“. ${ }^{1}$ Die Stoppuhr verkörperte einen aufgezwungenen Arbeitsrhythmus, ein neues Zeitbewusstsein, das sich durch Geschwindigkeit manifestierte. Schnelldampfer, Schnellzug, Tempo-Taschentücher und Kurzschrift sind nur einige neue Begriffe und Produkte, die sich in den 1920er Jahren herausbildeten und dieses Zeitbewusstsein kennzeichnen. ${ }^{2} 1927$ schuf John Heartfield die Collage „Ein Gespenst geht um in Europa" (Abb. 1), die eindringlich die negativen Verän-

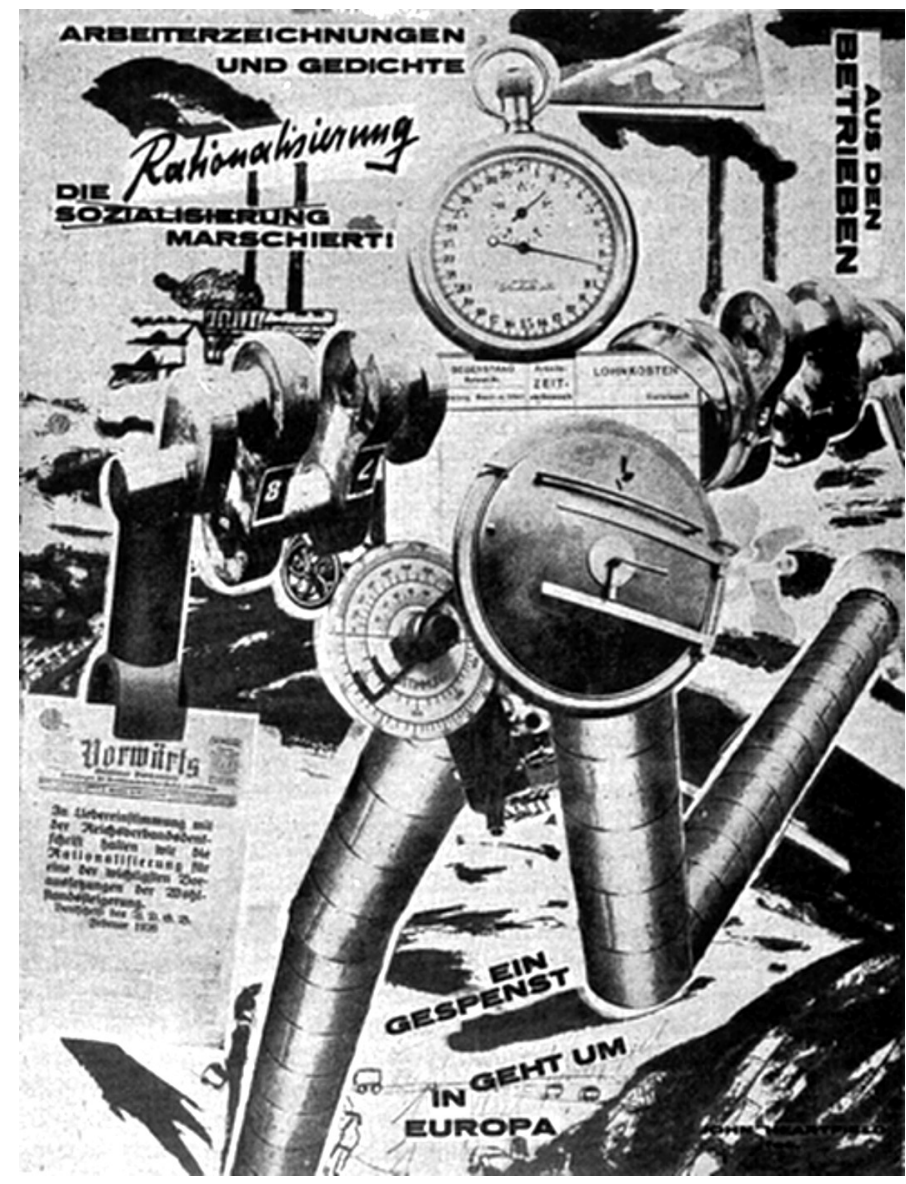

Abb. 1: Fotomontage von John Heartfield: Ein Gespenst geht um in Europa (1927). Quelle: Akademie der Künste zu Berlin (Hg.), John Heartfield (Ausstellungskatalog), Köln 1991, Abb. 47a.

1 Wolfgang König, Auf dem Weg in die Konsumgesellschaft (Technik und Gesellschaft. Historische Grundlagen der modernen Technik, Studienbrief), Tübingen 1993, S. 21.

2 Peter Borscheid, Der Tempomacher. Die Rationalisierung und die Beschleunigung des Lebens in den Weimarer Jahren, in: Zeitschrift für Unternehmensgeschichte 41, 1996, S. 125-138, bes. S. $128 \mathrm{f}$. 
derungen durch die Stoppuhr festhält. Den Kopf des maschinisierten Menschen bildet die Stoppuhr - als markanter Mittelpunkt, der alle umgebenden Bildfragmente vereinigt: Lohnkosten, Arbeitszeit, Rationalisierung und Maschinen. Durch die Stoppuhr wird aus dem Individuum eine seelenlose Arbeitsmaschine.

Die Uhr und das Uhrwerk waren schon seit ihrer Erfindung zu Beginn des 14. Jahrhunderts stets Träger übergeordneter Botschaften. Die Uhr und ihr gleichmäßig ablaufendes Räderwerk standen als Äquivalent für Mäßigung, Kontrolle und Fleiß. ${ }^{3}$ Der Arbeitgeber nutzte die Uhr, um die Arbeitskräfte und den Produktionsprozess zu koordinieren, und die Uhrzeit setzte sich als das reglementierende Prinzip des Arbeitsprozesses durch: „Die Uhrenkultur und das industrielle System reisten gemeinsam in die Zukunft, zwei untrennbar verbundene Kräfte, die sich gegenseitig definieren und formen halfen." ${ }^{* 4}$

Anliegen des folgenden Beitrags ist es, die Entstehungs- und Verwendungsgeschichte der Stoppuhr darzustellen und dabei besonders ihre Entwicklung zum Disziplinierungsinstrument der Industriearbeit in der Rationalisierungsbewegung des frühen 20. Jahrhunderts in den Blick zu nehmen. Es werden zuerst die Entstehungsgeschichte der Stoppuhr von ihren ersten Anfängen im 16. Jahrhundert bis zum Beginn des 20. Jahrhunderts und die Ursprünge ihrer Verwendung im Sport und beim Militär betrachtet. Danach wird die Funktion der Stoppuhr für die Taylorisierung der Industriearbeit erläutert und ihre Anwendung in der deutschen Rationalisierungsbewegung analysiert. Wie die Uhrenbauer ihre Produkte den neuen Anforderungen anpassten und damit gleichzeitig die Durchführung von Zeitstudien prägten, ist Gegenstand des letzten Abschnitts.

\section{Die Entwicklung der Stoppuhr vom 16. bis zum frühen 20. Jahrhundert}

Die Stoppuhr ist ein Kurzzeitmesser und als solche bezeichnet man im heutigen Sprachgebrauch Messinstrumente, die nur sehr kurze Zeitintervalle erfassen, im Gegensatz zu den die Tageszeit anzeigenden Uhren geben Stoppuhren oder Kurzzeitmesser Zeitabschnitte an, die nicht an die kontinuierlich ablaufende Tageszeit gebunden sind. Die kontinuierliche Tageszeit wird als das gesellschaftlich anerkannte Koordinierungsmittel aller zeitlichen Abläufe in Stunden, Minuten und Sekunden verstanden. Ein Kurzzeitmesser zeigt nur einen Ausschnitt dieses Zeitreglements. Der Mechanismus des „Anhaltens" der Uhr macht einen Kurzzeitmesser zur Stoppuhr. Zeigen die ersten

3 Otto Mayr, Uhrwerk und Waage. Autorität, Freiheit und technische Systeme in der frühen Neuzeit, München 1978; Klaus Maurice u. Otto Mayr, Die Welt als Uhr. Deutsche Uhren und Automaten 1550-1650, Berlin u. München 1980; Gerhard Dohrn-van Rossum, Die Geschichte der Stunde. Uhren und moderne Zeitordnungen, München u. Wien 1992.

4 Jeremy Rifkin, Uhrwerk Universum. Die Zeit als Grundkonflikt des Menschen, München 1988, S. 147. 
Stoppuhren Intervalle von einer Sekunde, so entwickelt sich die Zeitunterteilung auf Kurzzeitmessern bis hin zur Tausendstelsekunde.

Stoppuhren werden den zählenden Zeitmessinstrumenten zugeordnet, da sie die Zeiteinheiten der Dauer eines Vorganges erfassen, dessen Beginn und Ende durch eine Betätigung am Zähler abgegrenzt wird. ${ }^{5}$ Uhren, die zusätzlich zur Tageszeitanzeige einen Stoppmechanismus besitzen, werden heute nicht mehr zu den Kurzzeitmessern gezählt. Stoppuhren sollen also Zeitabschnitte messen oder festhalten, die sich zwar an der Tageszeit orientieren, aber eigentlich keinen Zusammenhang mit der Zeitfolge an sich aufweisen. Diese Zeitabschnitte sind im Gegensatz zur Uhrzeit geographisch unabhängig.

Die Stoppuhr hat sich aus einfachen Uhren mit Sekundenzeiger entwickelt. Die älteste bekannte Uhr dieser Art wird auf die Zeit zwischen 1560 und 1570 datiert. ${ }^{6}$ Uhren dieser Art besitzen zusätzlich zur Zeitanzeige eine „kleine Sekunde“. Der Sekundenzeiger sitzt auf dem Rad, das sich einmal in der Minute dreht. Noch hat die Sekunde in der Lebens- und Arbeitszeit keine Bedeutung, solche Uhren sind als technische Spielerei und als Statussymbol anzusehen. Nur bei astronomischen Beobachtungen spielte sie schon frühzeitig eine wichtige Rolle und ihre exakte Wiedergabe trieb die Entwicklung genauer Zeitanzeiger voran. Um 1581 verwendete z.B. Tycho Brahe ${ }^{7}$ zur Beobachtung eines Sterndurchganges durch die Mittagslinie eine Uhr mit Sekundenangabe. Allerdings beklagte er sich schon einige Jahre später über die Ungenauigkeit dieser Uhren. ${ }^{8}$ Auch andere große Astronomen wie Jost Bürgi (15521632 ) versuchten Uhren mit exakten Sekundenangaben zu bauen. ${ }^{9}$ Dies waren aber immer ortsfeste Uhren. Tragbare Uhren oder Taschenuhren mit zum Teil stoppbarem Sekundenzeiger sind erst aus dem 17. Jahrhundert überliefert. Möglich wurde dies durch die von Christiaan Huygens 1675 erfundene Unruhspirale. ${ }^{10}$

Diese alten Sekundenuhren werden heute meist unter dem Begriff „Doctor-Uhren“ (Abb. 2) zusammengefasst. Sie bleiben weiterhin Einzelstücke,

5 Weitere Zeitmessgeräte werden als schreibend, anzeigend oder schaltend bezeichnet. Vgl. Kurt Poppe (Bearb.), Zeitmeßgeräte für die Industrie, hg. v. Energie- und Betriebswirtschaftsstelle des Vereins Deutscher Eisenhüttenleute, Düsseldorf 1955, S. 1ff.; Klaus Heinz, Geräte zur Zeitmessung bei Arbeits- und Zeitstudien und ihre Einsatzmöglichkeiten, Köln 1967, S. 6-10.

6 Eine Abbildung der so genannten Orpheusuhr befindet sich in Philip G. Coole und Erwin Neumann, The Orpheus Clocks, London 1972, Plate 3.

7 1546-1601, dänischer Astronom, baute bis 1580 auf der Insel Hveen eine bedeutende Sternwarte „Uranienburg“. 1599 Umsiedlung nach Prag als Hofastronom Kaiser Rudolf II.

8 Ernst Zinner, Deutsche und Niederländische Astronomische Instrumente des 11.-18. Jahrhunderts, München 1957, S. 22. Es sind keine genaueren Angaben zu diesen „SekundenUhren" überliefert.

9 Ebd., S. 22 u. 268ff.; Jürgen Abeler, Ullstein Uhrenbuch. Eine Kulturgeschichte der Zeitmessung, Frankfurt a.M. u. Berlin 1994, S. 115.

10 Zur Unruhspirale siehe Reinhard Meis, Taschenuhren. Von der Halsuhr bis zum Turbillon, München 1999, S. 10f. 


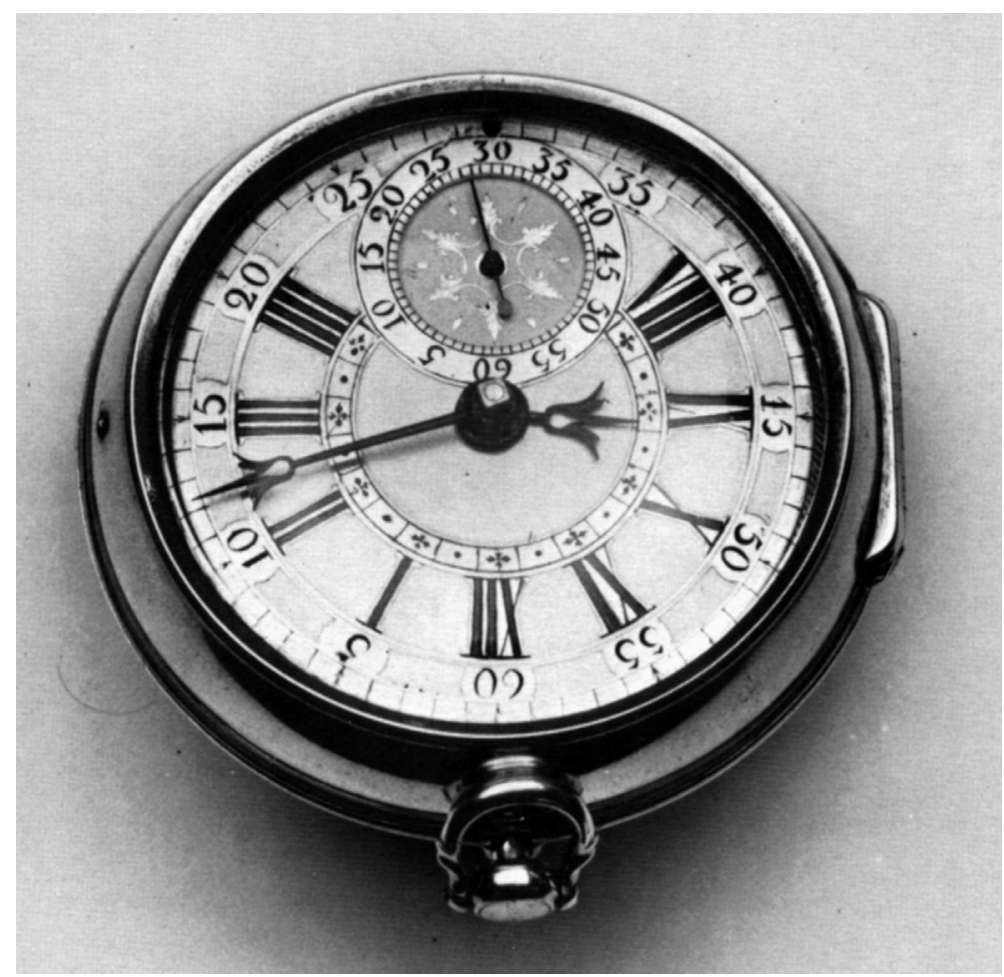

Abb. 2: „Doctor-Uhr“ ca. 1690. B. Bell Taschenuhr mit kleiner Sekunde. Quelle: Cedric Jagger, Wunderwerk Uhr, Zürich 1977, S. 130.

wie ein Fachbuch von 1774 vermerkt: ,eine Secundenuhr wird sich so leicht niemand, als ein Sternseher, oder Mathematiker, oder Arzt anschaffen; die beyden ersten zum mathematischen Gebrauche, und der letztere zu Beobachtung des Pulses. Denn außer dem mathematischen und medicinischen Gebrauche ist die Secundenzeigung auf den Taschenuhren überflüssig." ${ }^{11}$

Der Stoppmechanismus wurde sehr einfach gelöst. Ein kleiner Hebel am Gehäuserand schob entweder einen kleinen Stift in das Räderwerk und hielt es damit an, oder eine kleine Feder legte sich an die Unruhe und stoppte somit die Bewegung der Uhr. Mit dem Anhalten des Sekundenzeigers wurde gleichzeitig das gesamte Uhrwerk gestoppt und musste nach dem Ende einer Messung neu gestellt werden. Diese Uhren wurden hauptsächlich dazu verwendet, eine gemessene Zeit zu einer ortsfesten präzise laufenden Uhr zu transportieren. ${ }^{12}$ So wurden etwa Standuhren in Sternwarten mit Hilfe einer stoppbaren Sekundenuhr eingestellt. Bis in das 20. Jahrhundert hinein wurde die Ortszeit durch die Beobachtung des Sonnendurchgangs durch die Mit-

11 Zit. nach Christian Friedrich Vogel, Praktischer Unterricht von Taschenuhren, Leipzig 1774, Neudruck 1975, S. 6f.

12 Johann Wenzel, The „Doctors“ Stop Watch, in: Antiquarian Horology 17, 1987, S. 67 u. 77. 
tagslinie (Ortsmeridian) bestimmt. Die Stoppuhr wurde auf zwölf $\mathrm{Uhr}^{13}$ gestellt und angehalten. Beobachtete der Astronom den Sonnendurchgang, so betätigte er im Moment der Passage die Taschenuhr und konnte somit die genaue Ortszeit auf die Standuhr, die wesentlich genauer und auch länger als eine Taschenuhr lief, übertragen. ${ }^{14}$ Die tragbare Stoppuhr wurde auch Borduhr genannt und an Bord von Schiffen verwendet. Die Zeit konnte, durch eine astronomische Messung festgelegt und mithilfe einer transportablen Uhr zu einer ortsfesten sekundengenauen Uhr, dem Seechronometer, getragen werden. ${ }^{15}$

Im 18. Jahrhundert gab es zahlreiche technische Weiterentwicklungen des Uhrenmechanismus und auch des Stoppuhrmechanismus. 1757 erfand der Uhrmacher Thomas Mudge (1715-1794) die erste „freie“ Ankerhemmung, die eine reibungsarme Schwingung des Taktgebers ermöglichte. ${ }^{16}$

Als erste Stoppuhr, bei der Anfang und Ende eines Zeitabschnittes festgehalten werden konnte, wird der Sekundenchronograph von Rieussec aus dem Jahr 1821 bezeichnet. Der Begriff Chronograph (Zeitaufzeichnung) ist hier richtig gewählt, denn Beginn und Ende eines Messvorganges wurden als Tintenpunkte auf dem Zifferblatt festgehalten. Zusätzlich konnte die Uhr z.B. auf der Position „Null“ angehalten werden. ${ }^{17}$

Dennoch blieben die Stoppuhren unbefriedigend, denn der Sekundenzeiger ließ sich nicht exakt auf „Null“ zurücksetzen. Dieses im Rahmen der Uhrenentwicklung simpel erscheinende technische Problem wurde 1861 von Henry Piguet gelöst und 1862 von der schweizerischen Uhrenfirma Nicole \& Capt. auf der Weltausstellung in London vorgeführt. ${ }^{18}$ In der Folge nahmen mehrere Betriebe die Produktion von Stoppuhren auf. Um 1880 begann auch Moritz Grassmann, Uhrenfabrikant in Glashütte, einfache und preiswerte Stoppuhren herzustellen. „Nach diesen einfachen Sekundenzählern entstand sehr bald eine sehr lebhafte Nachfrage, und um dieser nachzukom-

13 Jede gute englische Standuhr ab 1680 wurde mit einer Äquationstabelle für ihren Standort geliefert. Wurde nun also der Durchgang der Sonne durch die Mittagslinie beobachtet, dann stand die Stoppuhr nicht auf zwölf Uhr, sondern an der dem Datum entsprechenden mittleren Zeit. Vgl. hierzu Johann Wenzel, Warum haben Uhren Sekundenzeiger? in: Alte Uhren, 1980, H. 1, S. 38-42; Wilhelm Meyer (Bearb.), Diesterwegs Populäre Himmelskunde und mathematische Geographie, Berlin 1890, S. 289-295.

14 Vgl. Wenzel (wie Anm. 13), S. 67.

15 Die Genauigkeit dieser Uhren war von extremer Wichtigkeit, da zwei Zeitsekunden etwa einem Kilometer auf dem Äquator entsprechen und eine Fehlweisung der Uhr um mehrere Sekunden die Standortbestimmung erheblich ungenauer ausfallen lassen würde. Wenzel (wie Anm. 13), S. 41f.

16 Nähere Erläuterungen zu dieser Hemmung wie auch zu den anderen Hemmungssystemen Meis (wie Anm. 10), S. 12-29.

17 Eine genaue Funktionsbeschreibung und Werkzeichnung dieser Uhr findet sich in Emanuel Schreiber, Vollständiges Handbuch der Uhrmacherkunst, Weimar 1865, S. 501-509.

18 Adolphe Chapiro, Taschenuhren aus vier Jahrhunderten. Die Geschichte der französischen Taschenuhren, München 1995, S. 394. 
men, leitete ich die Erzeugung derselben in grösserem Maasstabe ein, nachdem ich mir das Recht daran durch Patente gesichert hatte", schrieb er $1905 .{ }^{19}$ Den Nutzen dieser Uhren sah er besonders für Ingenieure, Mediziner und im Sport und entwickelte dementsprechend vier unterschiedliche Typen von Stoppuhren. Über die Zifferblattanzeige ist leider nichts zu erfahren, doch es ist anzunehmen, dass sie eine Sechzigstel-Teilung besaß ${ }^{20}$ Die in Abbildung 3 dar-

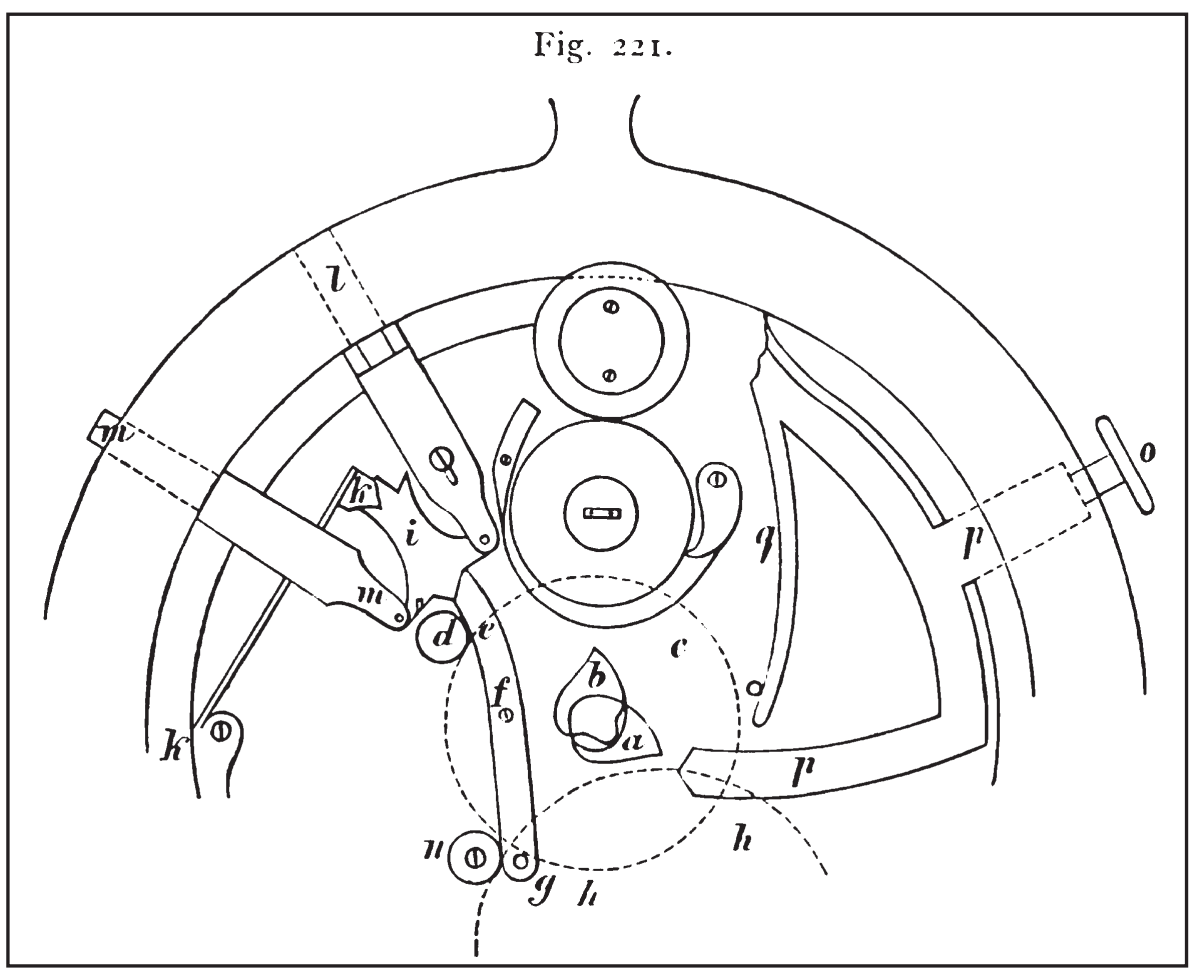

Abb. 3: Einfacher Sekundenzähler von Grossmann (D.R.P. 1878). Quelle: Eugen Gelcich, Die Uhrmacherkunst und die Behandlung von Präcisionsuhren. Handbuch für Uhrmacher, Wien, Pest u. Leipzig 1892, S. 575. $l, m$ und $o$ bezeichnen Drücker, die das Starten, Stoppen und Rücksetzen auf Null des Sekundenzeigers bewirken. Damit der Beobachter sich nicht auf die Wahl der Drücker konzentrieren muss, bleibt der gerade verwendete Drücker $l$ flach am Gehäuserand liegen und der zweite Drücker $m$ tritt stark hervor. Der Mechanismus zum Stoppen der Unruhe $h$ wird durch die Hebel $e, f$ und $g$ dargestellt. Das untere Ende des Hebels trägt einen kleinen Stift $g$, der durch Verschieben des Hebels (durch Druck auf $l$ oder $m$ ) auf eine kleine Schraube mit abgeschrägtem Kopf $n$ hinauf oder herab geschoben wird. In der dargestellten Position wird die Unruh durch den kleinen Stift $g$ angehalten. Die Nullstellung erfolgt durch den Drücker $o$. Wird er gedrückt, dann schiebt sich der Hebel $p$ an die beiden Herzscheiben $a$ und $b$ bis diese mit ihren flachen Seiten am Hebel anliegen. Da diese direkt mit dem Sekunden- und mit dem Minutenzeiger verbunden sind, nehmen sie die gewünschte Null-Position ein.

19 Zitiert nach Claudius Saunier, Lehrbuch der Uhrmacherei in Theorie und Praxis, Bd. 3, Bautzen 1905, Reprint Leipzig 1979, S. 294.

20 Ebd., S. $295 f$. 
gestellte Mechanik dürfte der Stoppuhr-Technik zu Beginn des 20. Jahrhunderts entsprechen.

Die Bandbreite der Stoppuhren vergrößerte sich zunehmend. Erhöhte Nachfrage für Sport, Militär und Wissenschaft, sowie schon vorhandene moderne Produktionsmethoden in der Uhrenindustrie ermöglichten die Herstellung unterschiedlichster Stoppuhrtypen. ${ }^{21}$ Der nächste Schritt zur preiswerten und allgemein benutzten Stoppuhr war die industrielle Serienproduktion, die schon 1845 von der Firma Vacheron und Constantin in der Schweiz mit Spezialmaschinen begonnen wurde. ${ }^{22}$ Die folgenden beiden Abschnitte beschäftigen sich mit der Verwendung der Stoppuhr im Sport und beim Militär. In beiden Verwendungsarten sind Vorläufer für die Nutzung der Stoppuhr bei der Rationalisierung von Arbeitsprozessen zu erkennen.

\section{Die Anwendung der Stoppuhr im Sport}

Die Entwicklung der Kurzzeitmessung ist untrennbar mit dem Rekord- und Leistungsgedanken von sportlichen Aktivitäten verbunden. Die Zeitregistrierung ermöglichte es, unabhängig von Zeit und Ort einen Wettkampf zu gewinnen, der nun nicht mehr gegen einen gegnerischen Athleten geführt werden musste, sondern gegen die von ihm geleistete „Bestzeit“. ${ }^{23}$

Die Messung einer zeitlichen Dauer von Wettkämpfen hat ihren Ursprung im englischen Adelssport, den „Matches against Time“, die Ende des 16. Jahrhunderts modern wurden. Die ersten Wetten dieser Art bezogen sich noch auf lange Zeiträume über mehrere Tage..$^{24}$ Ungefähr ab 1731 wurden Pferdewetten und ihre Ergebnisse, gemessen in Stunden, Minuten und Sekunden, in Zeitungen veröffentlicht. ${ }^{25}$

1898 begann die olympische Bewegung mit der Aufstellung normierter Wettkampfregeln unter Einbeziehung gemessener Zeiteinheiten. ${ }^{26}$ Trotz der frühen Entwicklung der „Sekundenuhren“ wurde das Spektrum der Möglichkeiten, das die Uhr zur Leistungsbewertung anbot, erst seit der Mitte des 19. Jahrhunderts ausgeschöpft. Jahrhunderte vorher wurden Wettkämpfe, insbe-

211785 produzierte die Firma Japys in der Schweiz bereits Taschenuhr-Rohwerke. Das sind komplette Uhrwerke, die noch nicht qualitativ bearbeitet wurden. Die Endbearbeitung fand in der Werkstatt einzelner Uhrmacher oder kleiner Firmen statt. Ab diesem Zeitpunkt wurden auch die ersten Spezialmaschinen zur seriellen Produktion von Uhrwerken entwickelt. Vgl. Chapiro (wie Anm. 18), S. 270.

22 Zur Firmengeschichte von Vacheron und Constantin siehe Helmut Mann, Vacheron und Constantin, in: Alte Uhren und moderne Zeitmessung, Bd. 3, 1986.

23 Henning Eichberg, Leistung, Spannung, Geschwindigkeit, Sport und Tanz im gesellschaftlichen Wandel des 18./19. Jahrhunderts (Stuttgarter Beiträge zur Geschichte und Politik, Bd. 12), Stuttgart 1978, S. 58f.

24 Zur Geschichte der „Matches against Time“ siehe Maria Kloeren, Sport und Rekord. Kultursoziologische Untersuchungen zum England des sechzehnten bis achzehnten Jahrhunderts, Diss. 1935, (Sport: Kultur, Veränderung, Bd. 9), Münster 1985.

25 Ebd., S. 214

26 Eichberg (wie Anm. 23), S. 58f. 
sondere Pferderennen, zum Anlass einer Geldwette oder als reines Vergnügungs-Spektakel verstanden. Sportliche Höchstleistungen, wie etwa Gewichtheben oder Turnen waren im Bereich des Jahrmarktes und somit der Kuriositäten angesiedelt. Erst mit dem Entstehen der Turnvereine Anfang des 19. Jahrhunderts wird die Höchstleistung aus dem Kuriosum heraus in den Allgemeinsport verlagert. Ab diesem Zeitpunkt wird es auch üblicher, Bestleistungen bei Wettläufen mit der Stoppuhr festzuhalten und sie durch das Führen von Leistungslisten vergleichbar zu machen. ${ }^{27}$

$\mathrm{Zu}$ Beginn des 20. Jahrhunderts hatte der Gewinn auf Zeit alle Sportbereiche erfasst. Besonders für den Automobil- und Segelsport wurden neue Zifferblattanzeigen entwickelt, beispielsweise so genannte "Sportsignaluhren“" und Stoppuhren mit Tachymeteranzeige. Wichtig hierbei ist die Markierung einer Distanz innerhalb eines zeitlichen Rahmens. ${ }^{28}$

Zusätzlich zur Messung einer Bestleistung finden sich im Sport Ansätze, eine Bewegung in einzelne Bewegungssequenzen zu zergliedern. Schon in den ständischen Exerzitien des 17. Jahrhunderts wurden beim Voltigieren, Fechten und Figurenreiten Bewegungssegmente klassifiziert und einzeln eingeübt. Diese Aufteilung der Bewegung in Einzelsegmente wurde Anfang des 19. Jahrhunderts für gymnastische Übungen weiterentwickelt. Die Bewegungen wurden separat trainiert und wahlweise zu verschiedenen Bewegungskombinationen zusammengesetzt. ${ }^{29}$ Die Zergliederung einer Bewegung führte im 20. Jahrhundert schließlich zum Leistungstraining, bei welchem durch exaktere Zeitmessungen und Filmaufnahmen die Einzelbewegung immer weiter optimiert und effektiver gestaltet wurde.

\section{Militärische Verwendung der Stoppuhr}

Ein wesentliches Element militärischer Taktik ist die zeitliche Abstimmung der Bewegung des einzelnen Soldaten mit der Bewegung des gesamten Heeres. Gerade mit dem Einsatz des Gewehres mussten alle einzelnen Bewegungen kalkulierbar werden. ${ }^{30}$ Dass diese Vorstellungen in die Praxis umgesetzt und auch von Zeitgenossen erkannt wurden, lässt sich einem Exerzier-

27 Vgl. Eichberg (wie Anm. 23), S. 58f., siehe auch S. 54f. die Entstehung des auf Leistungssteigerung beruhenden Sportkonzeptes durch den Gymnastiklehrer Guts Muths. Er hat die Leistung seiner Zöglinge mit einer Stoppuhr auf Viertelminuten genau bemessen und protokolliert. Die Leistungsvergleiche sollten die Schüler motivieren und anspornen, die Leistung zu steigern.

28 Vgl. M. Loeske, Die Chronographen und Stoppuhren für besondere Zwecke, in: Deutsche Uhrmacherzeitung 45, 1921, H. 1, S. 4-7 u. H. 3, S. 31 f.

29 Vgl. Eichberg (wie Anm. 23), S. 51-60, 129 u. 134f.

30 Jürgen P. Rinderspacher, Gesellschaft ohne Zeit. Individuelle Zeitverwendung und soziale Organisation der Arbeit, Frankfurt a.M. 1985, S. 44. Rinderspacher erwähnt einschneidende Veränderungen im Militärwesen durch die Erfindung des Gewehrs. Gemeint ist die Einführung des Ladestockes um 1730, mit dessen Hilfe Friedrich der Große eine Feuergeschwindigkeit von fünf Schuss in der Minute erreichte. Vgl. Meyers KonversationsLexikon, Leipzig 1897, „Handfeuerwaffen“, Bd. 8, S. $316 \mathrm{f}$. 
reglement von 1766 entnehmen. Hier wurde nicht nur die Dauer der einzelnen Handgriffe in Sekunden gemessen: „Abgestimmt wurden die Handhabung, sowie das Laden und Abfeuern eines Gewehres mit dem Exerzieren einzelner Griffelemente und Bewegungen, die in verschiedene Zeiten aufgeteilt wurden“. Auch die Schrittdauer wurde in Sekunden vorgegeben. „Was die Dauer anbelangt, so hat man für den kleinen Schritt und für den gewöhnlichen Schritt eine Sekunde, für den doppelten Schritt eine halbe Sekunde, für den Straßenschritt etwas mehr als eine Sekunde. Den Schrägschritt macht man ebenfalls in einer Sekunde". ${ }^{31}$ Die Sekundenangaben weisen auf die Verwendung der Sekundenuhr hin, so dass angenommen werden kann, dass auch die Bewegungselemente der Schießregel nach exakten Zeiten vorgegeben wurden. Zur Kontrolle der Schrittzeiten wurden um 1800 so genannte Schrittzähler (Abb. 4) verwendet. Sie zeigen die Schrittdauer pro Minute entweder optisch durch den Zeigersprung, oder akustisch durch einen taktgebundenen Schlag auf eine Tonfeder an. Die Taktvorgabe reichte meist von 60 bis 120 Schlägen pro Minute und konnte variabel eingestellt werden. ${ }^{32}$

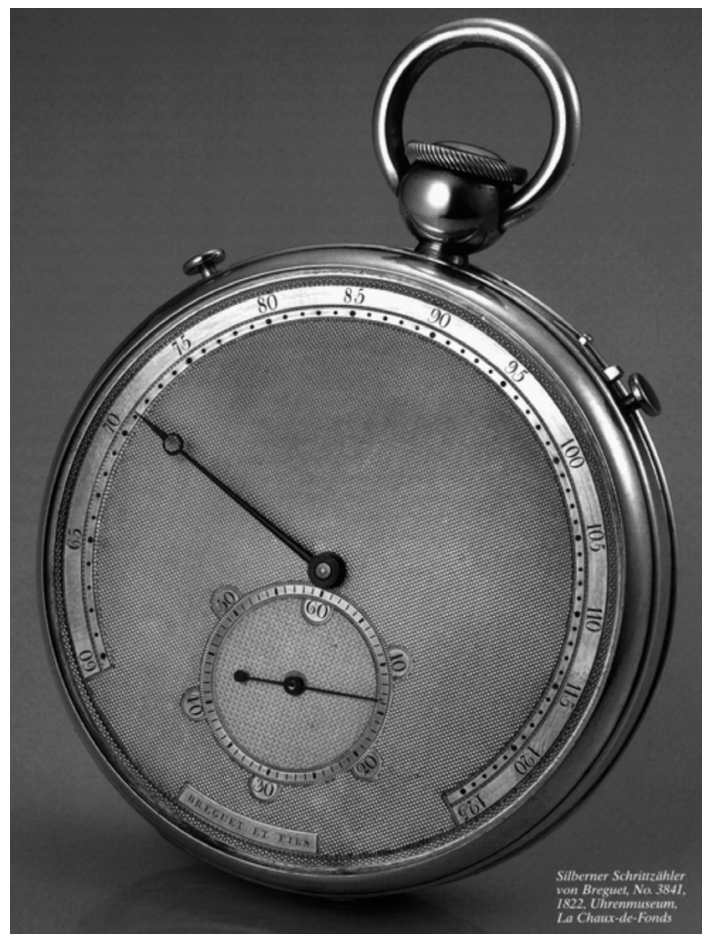

Abb. 4: Silberner Schrittzähler von Louis Breguet. Quelle: Wilhelm Schultz, Im Gleichschritt, in: Klassik Uhren 21, 1998, H. 2, S. 10. Foto: Collections du Musée international d'horlogerie, La Chaux-de-Fonds, Suisse.

31 Ordonnance du janvier 1766, pou regler l'exercise de l'infanterie, zitiert nach Michael Foucault, Überwachen und Strafen. Die Geburt des Gefängnisses, Frankfurt a.M. 1994, S. 194ff.

32 Der Mechanismus dieser Uhren ist ausführlich erläutert in Wilhelm Schultz, Im Gleichschritt, in: Klassik Uhren 21, 1998, H. 2, S. 10-15. 
Im Rückblick wurden in den militärischen Exerzierübungen wichtige Vorläufer für ein neues Zeitreglement in der Rationalisierungsbewegung gesehen: „Wenn Sie sich ins Gedächtnis zurückrufen, wie in der Schießvorschrift zunächst gehirnmäßig der ganze Vorgang des Schießens in die kleinste Einzelheit zerlegt wird, wie darin jede Handbewegung, ja, jede Fingerbewegung, das Krümmen des Zeigefingers, sodann das Schließen des Auges usw. in ganz eindeutiger Weise vorher festgelegt wird, so werden Sie mir zugeben, daß das nichts weiter ist als Scientific Management."“33

Mit der Entwicklung präziserer Waffen wurde die Stoppuhr beim Militär auch im Bereich der Entfernungsmessung und der Ballistik verwendet. Bei Übungen und im Kriegsfall konnte mit ihrer Hilfe die Entfernung gegnerischer Geschütze bestimmt werden. Die Stoppuhr steht in der Nullposition und wird bei der Beobachtung des Mündungsfeuers gestartet. Erreicht der Schall den Beobachter, so wird die Uhr gestoppt. Aus der bekannten Schallgeschwindigkeit (ca. 333 Meter in der Sekunde) und der abgelesenen Zeitspanne lässt sich die Entfernung berechnen. Damit lässt sich umgekehrt auch das eigene Geschütz auf die gegnerische Position richten. Hierfür wurden wiederum genaue Kenntnisse der spezifischen Leistung der Waffen benötigt. Diese wurden in praktischen Versuchsreihen mit Hilfe von Kurzzeitmessern ermittelt und auf einer Schusstafel festgehalten. Sie beinhaltete unter anderem die Daten für verschiedene Geschosse, Pulverladungen und Schussfolgen, aufgeschlüsselt nach Höhenrichtung, Fallwinkel, Flugzeit und Geschossgeschwindigkeit. ${ }^{34}$

Wie bei vielen anderen technischen Instrumenten sind auch bei der Stoppuhr Entwicklung und Verwendung nicht immer eindeutig bestimmten Gebieten oder Begriffen zuzuordnen. In der Experimentalphysik, der Ballistik, der Astronomie, dem Militär und sogar in der Verhaltensforschung fanden ähnliche oder gleiche Kurzzeitmesser Verwendung. In diesen Bereichen wurde eine Genauigkeit der Messergebnisse bis auf den Tausendsten Teil einer Sekunde gefordert. Die 1862 in Berlin gegründete Firma Loebner spezialisierte sich auf diese Anforderungen. Sie bot z.B. Uhren mit Tausendstelsekunden zum Messen von Geschossgeschwindigkeiten, aber auch Uhren für Pferderennen und Tertienuhren für Torpedoschießen an. ${ }^{35}$

33 Conrad Matschoß, in seinem Diskussionsbeitrag auf der 45. Hauptversammlung des VDI 1913 in Leipzig, in: Technik und Wirtschaft 6, 1913, H. 8, S. 552.

34 Otto Lueger (Hg.), Lexikon der gesamten Technik und ihrer Hilfswissenschaften, Bd. 1: Ballistik, Stuttgart u. Leipzig 1914, S. 535f.

35 o.A., Jubiläum, 40 Jahre Firma Loebner, in: Deutsche Uhrmacherzeitung 26, 1902, Nr. 20, S. 331. Vgl. auch Johann Wenzel, Ältere Uhren und Geräte zum Messen von Sekundenbruchteilen, in: Klassik Uhren 18, 1995, S. 39-50, hier S. 45f. Tertie ist der sechzigste Teil einer Sekunde. 


\section{Der Einsatz der Stoppuhr im Arbeitsprozess}

$\mathrm{Zu}$ Beginn der 1920er Jahre hatten sich eindeutig verschiedene Formen der Kurzzeitmessung und entsprechend unterschiedliche Uhrentypen herauskristallisiert. Das Gebiet umfasste zum einen Spezialuhren, wie sie die Firma Loebner herstellte, und zum anderen die Stoppuhren für den Breitensport, den privaten Gebrauch, für wissenschaftliche Messungen (die mit dem Auge noch zu verfolgen waren) und für die Industrie. Die Stoppuhr war zum Zeitpunkt ihrer umfassenden Nutzung zu Rationalisierungszwecken im Zuge der Rationalisierungsbewegung ${ }^{36}$ bereits ein technisch ausgereiftes und vielseitig verwendetes Messinstrument.

Das Rationalisierungsbündnis, das als Bewegung bezeichnet wurde, weil es verschiedenste Akteure aus der Industrie und staatliche Institutionen vernetzte, die zur Ankurbelung des Rationalisierungsgedankens zusammenwirkten ${ }^{37}$ konnte, außer auf die schon vorhandenen technischen Möglichkeiten der Stoppuhr, in erster Linie auf die theoretischen Arbeiten von Frederick Winslow Taylor zurückgreifen. In Taylors Werk wurde erstmals der Uhr als wissenschaftlichem Messinstrument im Arbeitsprozess die führende Rolle zugewiesen. Die von ihm veröffentlichten Schriften ${ }^{38}$ zur wissenschaftlichen Betriebsführung benennen die Stoppuhr als unabdingbares Mittel zur Erfassung einer exakten und neutralen Größe, der Zeit, auf der die Vorkalkulation, die Entlohnung, Arbeitsvorgaben und Kontrolle der Leistung basieren sollten. Vor allem infolge der deutschen Übersetzungen ${ }^{39}$ wurden in den Technischen Vereinen, wie etwa dem VDI, und insbesondere in der Metallindustrie die Zeitstudien Taylors intensiv wahrgenommen und in den Zeitschriften diskutiert. ${ }^{40}$ Die Zeitstudie war die wesentliche Neuerung, die die Erfassung von Arbeitsprozessen optimieren sollte.

36 Der zeitliche Rahmen der Rationalisierungsbewegung wird in der Literatur überwiegend auf die Jahre 1924 bis 1929 - zum Teil bis 1933 - eingegrenzt. Siehe hierzu Hans Albert Wulf, Maschinenstürmer sind wir keine. Technischer Fortschritt und sozialdemokratische Arbeiterbewegung, Frankfurt a.M. 1987; Günter Spur, Vom Wandel der industriellen Welt durch Werkzeugmaschinen, München 1991, S. 402; Thomas von Freyberg, Industrielle Rationalisierung in der Weimarer Republik, Frankfurt a.M. u. New York 1989.

37 Freyberg (wie Anm. 36), S. 302f.

38 Frederick Winslow Taylor, Shop Management, New York 1903; ders., The Principles of Scientific Management, New York 1911; ders., On the Art of Cutting Metal, New York 1906.

39 Frederick Winslow Taylor, Über Dreharbeit und Werkzeugstähle, dt. von Adolf Wallichs, Berlin 1908; Frederick Winslow Taylor, Die Betriebsleitung, insbesondere die Werkstätten, dt. von Adolf Wallichs, Berlin 1909; Frederick Winslow Taylor, Die Grundsätze wissenschaftlicher Betriebsführung, dt. von R. Roeseler, München 1913.

40 Siehe Heidrun Homburg, Rationalisierung und Industriearbeit. Arbeitsmarkt - Management - Arbeiterschaft im Siemens-Konzern Berlin 1900-1939 (Schriften der Historischen Kommission zu Berlin, Bd. 1), Berlin 1991, S. 293, Veröffentlichungen nach 1918, Anm. 133. Lothar Burchardt, Technischer Fortschritt und sozialer Wandel. Das Beispiel der Taylorismus-Rezeption, in: Wilhelm Treue (Hg.), Deutsche Technikgeschichte, Göttingen 1977, S. 52-98. 
Die Grundidee Taylors bestand vor allem in der Trennung von geistiger und körperlicher Arbeit. Noch ehe eine Arbeit begonnen wurde, sollte sie theoretisch und umfassend dokumentiert werden. ${ }^{41}$ Damit verband sich die Absicht, sowohl die noch bestehende Autonomie des qualifizierten Arbeiters im Produktionsprozess zu brechen als auch die Leistungsreserven der Arbeiter zu erschließen, um der von den Unternehmern angenommenen gemeinschaftlichen Leistungszurückhaltung entgegenzuwirken. Ein geeignetes Mittel schien zum einen, Maschinensysteme einzusetzen, die den Arbeiter austauschbar werden ließen, zum anderen ein System aus Regeln und Methoden einzuführen, das zuvor von der Betriebsleitung entwickelt worden war. ${ }^{42}$

Taylor ging davon aus, dass sich jede Arbeit, insbesondere die physische, in ganz genaue, einfache und kurze Bewegungsabläufe zerlegen lasse, deren Dauer mit Hilfe einer Stoppuhr ermittelt werden könne.$^{43}$ Der Vorteil, der für beide Seiten in dieser Verwissenschaftlichung des Arbeitsprozesses auf den ersten Blick zu erkennen ist, ist die Steuerung der Lohnpolitik über den Zeitfaktor. Die Berechnung der Leistung basierte nach Taylor auf dem Erreichen eines bestimmten Arbeitspensums, das mit Hilfe von Zeit- und Bewegungsstudien festgelegt wurde.

Gerade auf die Entlohnung richtete die Rationalisierungsbewegung ihr Hauptaugenmerk. Der Arbeiter wollte einen leistungsgerechten und vor allem sicheren Lohn, der Unternehmer brauchte einen kalkulierbaren Stückpreis. „Akkordlohn ist Mordlohn“ war das Schlagwort der Arbeiter bis weit in das 20. Jahrhundert hinein, Bummelei und Akkordfälschung das Gespenst der Unternehmer. ${ }^{44}$ Das neue Lohnsystem zielte darauf ab, einen Anreiz zur Intensivierung der Arbeit bei gleichzeitiger Verkürzung der Arbeitszeit zu setzen, und dem Unternehmer eine genaue Kostenkalkulation zu ermöglichen.

Voraussetzung einer Zeitstudie und auch einer genauen Vorkalkulation ist die Kenntnis der Maschinenleistung, der Maschinenzeiten sowie der Zeiten für Einrichten und Bestücken einer Maschine. Noch 1920 arbeitete zum Beispiel Siemens an einer Kartei über verwendete Werkzeugmaschinen. ${ }^{45}$

41 Taylor, Betriebsführung (wie Anm. 39), S. 37ff.

42 Werner Siebel, Fabrikarbeit und Rationalisierung, in: Jochen Boberg, Tilman Fichter u. Eckhart Gillen (Hg.), Exerzierfeld der Moderne. Industriekultur in Berlin im 19. Jahrhundert, München 1984, S. $317 \mathrm{f}$.

43 Frank Bunker Gilbreth, Das ABC der wissenschaftlichen Betriebsführung, nach dem Amerikanischen frei bearbeitet von Dr. Colin Ross, Berlin 1917, S. 2f.

44 Zum Schlagwort „Akkordlohn ist Mordlohn“ siehe Ludwig Bernhard, Die Akkordarbeit in Deutschland, Göttingen 1903, Vorwort, S. VI. Arbeiterstandpunkte zu Akkordlohn und Zeitlohn sind im Fragenkomplex: „Was ist ihnen lieber, Akkord oder Stundenlohn, und Warum?" bei Adolf Levenstein, Die Arbeiterfrage, München 1912, S. 33-39 nachzulesen. $\mathrm{Zu}$ Bummelei und Akkordverfälschung vgl. Alf Lüdtke, Arbeitsbeginn, Arbeitspause, Arbeitsende, in: Sozialgeschichte der Freizeit, Wuppertal 1982, S. 106. Vgl. ebenfalls Taylor, Betriebsleitung (wie Anm. 39), S. 9ff., §24-28.

45 Der Siemens-Konzern kann hier nur als ein Beispiel zitiert werden. Eine genaue Betrachtung der Herstellung und Verwendung der Maschinenkartei als eine der ersten zeitöko- 
Beruhte die Kenntnis der Maschinenleistung bisher auf Erfahrungen und Schätzungen der Facharbeiter oder Meister, sollte diese nun voll und ganz durch einheitliche Methoden und Richtwerte ersetzt werden. Die physische Arbeit wurde in ganz genaue, einfache und kurze Bewegungsabläufe untergliedert, deren Dauer mit Hilfe der Stoppuhr ermittelt wurde. ${ }^{46}$ Dies ermöglichte, die Bewegungen des Arbeiters sinnvoll zu kombinieren und zeitraubende Bewegungen zu verhindern. Die Aufnahme dieser Ist-Zeit konnte in einer Arbeitsunterweisungskarte festgehalten werden. Als wichtigste Stufe von Taylors Effizienzmaßnahmen folgte die Zeitstudie, mit deren Hilfe die schon rationalisierten Arbeitsabläufe in ihren Einzelbewegungen aufgenommen und als Normbewegung festgelegt wurden.

Damit waren alle Grundsteine gelegt ${ }^{47}$ welche die Rationalisierungsbewegung aufnahm und modifizierte. Frank Bunker Gilbreth und seine Frau Lillian Gilbreth entwickelten etwa für Studien über Bewegungsabläufe und Ermüdung ${ }^{48}$ einen eigenen Kurzzeitmesser, der Millionstelsekunden aufzeigte. Mit diesem konnte die von ihnen filmisch erfasste Bewegungsstudie in Einzelbewegungen zergliedert und die zeitliche Dauer erfasst werden ${ }^{49}$ Die ermittelten Zeiten wurden anschließend in der Werkstattsituation mit Hilfe der Stoppuhr nachgeprüft. ${ }^{50}$

Ein kurzer Blick auf die Psychotechnik ${ }^{51}$ kann zeigen, wie auch andere Forschungsgebiete die Entwicklung der Zeitstudie förderten und beeinfluss-

nomischen Maßnahmen findet sich bei: Homburg (wie Anm. 40), S. $451 \mathrm{ff}$. Vom Ausschuß für Maschinen- und Handarbeitsbestimmungen des AWF wurden 1922 Kartenentwürfevorgestellt, die die Erstellung und Handhabung einer Maschinenkartei vereinfachen sollten: „Unsere alten Kalkulationsmittel erwiesen sich als ungenügend. Man suchte nach neuen Hilfsmitteln, und als eines der wertvollsten hat man die Maschinenleistungskarte erkannt, da nur mittels dieser die genaue rechnerische Ermittlung der Maschinenzeiten möglich ist.“ Zit. nach Eifler, Maschinenkarten, in: Der Betrieb 4, 1922, H. 15, S. 93.

46 Gilbreth/Ross (wie Anm. 43), S. 2f.

47 Taylor, Betriebsleitung (wie Anm. 39), Kap. XII, Vornahme der Zeitstudien, S. 91-101.

48 Die Ermüdungsstudie als Teilaspekt der Arbeits- oder Bewegungsstudie bezieht sich nicht in erster Linie, wie der Name vermitteln könnte, auf die Regenerations- und Erholungszeit, die ein Arbeiter nach einer speziellen Arbeit benötigt, sondern auf die Ausmerzung ermüdender Bewegungen und Arbeitspositionen, denn ermüdende Bewegungen bedeuteten Verschwendung von Zeit und menschlicher Arbeitskraft. Siehe hierzu Frank Bunker Gilbreth, Ermüdungsstudium. Eine Einführung in das Gebiet des Bewegungsstudiums, Berlin 1921. Deutsche Übertragung: Irene Witte, S. 1. Zu Witte vgl. Rita Pokorny, Taylor - Gilbreth - Ford aus der Sicht der Rationalisierungsexpertin Irene Witte (1894-1976), in: Technikgeschichte 70, 2003, S. 153-183.

49 Gilbreth/Ross (wie Anm. 43), S. 4.

50 Ebd., S. 63.

51 Peter Hinrichs u. Lothar Peter, Industrieller Friede, Arbeitswissenschaft und Rationalisierung in der Weimarer Republik, Köln 1976, S. 35-42. Siehe auch Walter Moede, Die psychotechnische Arbeitsstudie, Richtlinien für die Praxis, in: Praktische Psychologie 1, 1920, H. 5, S. 136-146; Regina Weber, „Der rechte Mann am rechten Platz“. Psychotechnische Eignungsprüfungen und Rationalisierung der Arbeit bei Osram in den 20er Jahren, in: Technikgeschichte 68, 2001, S. 21-51. 
ten. Walter Moede ${ }^{52}$ sah die Grundzüge der Psychotechnik durch zwei Hauptaufgabengebiete gekennzeichnet, die Eignungsprüfung und die Rationalisierung der Arbeits- und Anlernverfahren. Die wichtigste Methode war dabei die experimentelle Arbeitsstudie. ${ }^{53}$

Die formale Arbeitstudie, wie sie von Taylor eingeführt wurde, maß den Zeitwert der Arbeit mit der Stoppuhr. Auch psychotechnische Eignungs- und Leistungstests wurden - ähnlich wie Zeit- und Bewegungsstudien - mit Hilfe von Stoppuhren oder ähnlichen Kurzzeitmessern gemacht, doch Uhren und Apparaturen wurden auch eigens für die industrielle Psychotechnik entwickelt. Hierzu gehört zum Beispiel eine Arbeitskontrolluhr von 1918 (Abb. 5). Der so genannte Arbeitspsychograph zeichnete alle ausgeführten Arbeiten und Pausen an einer Maschine ähnlich wie ein Fahrtenschreiber in einer Kurve auf. ${ }^{54}$

Parallel zur Taylorschen Zeitstudie wurde noch ein anderes Arbeitssystem in Deutschland eingeführt, das auch auf der Zerstückelung des Arbeitsprozesses und einer exakten Zeiterfassung mit der Stoppuhr basierte, die Fließbandarbeit. ${ }^{55}$ Fließarbeit ist insofern eine völlig andere Art des Umgangs mit den zerlegten Arbeitsprozessen als Taylor es vorschlug, da der Arbeiter nicht

Abb. 5: Arbeiterkontrolluhr. Quelle: Günther Burrichter, Die industrielle Psychotechnik und der durchsichtige Betrieb, in: Staatliche Kunsthalle Berlin und Neue Gesellschaft für Bildende Kunst (Hg.), Rationalisierung 1984, Berlin 1983, S. 75.

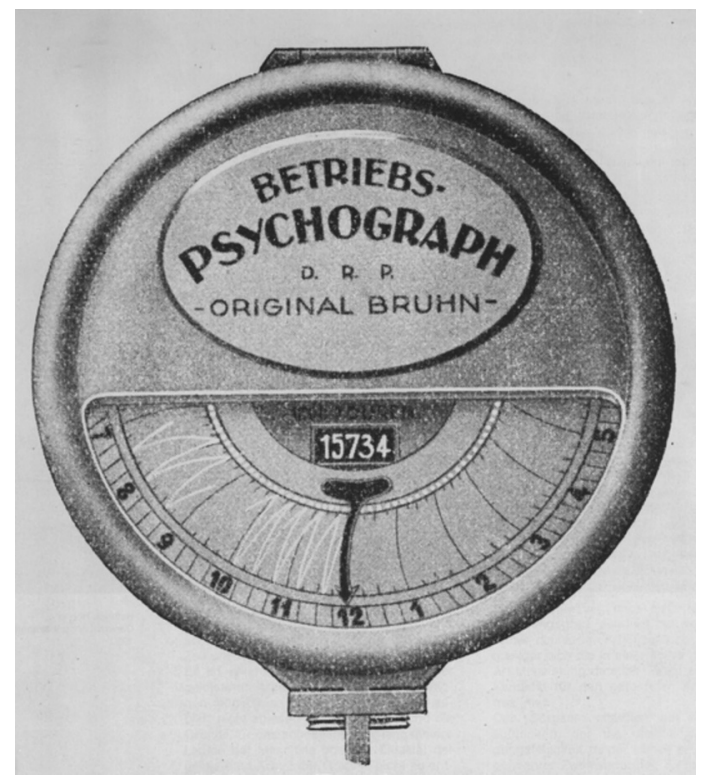

52 Walther Moede (1888-1958) war 1918 Leiter des Instituts für Psychotechnik an der Technischen Hochschule Berlin.

53 Moede (wie Anm. 51).

54 Günther Burrichter, Die industrielle Psychotechnik und der durchsichtige Betrieb, in: Staatliche Kunsthalle Berlin und Neue Gesellschaft für Bildende Kunst (Hg.), Rationalisierung 1984, Berlin 1983, S. 74f.

55 Zur Entwicklung der Fließfertigung siehe Sigfried Giedion, Die Herrschaft der Mechanisierung. Ein Beitrag zur anonymen Geschichte, Frankfurt a.M. 1982, S.109f. u. 140-143. 
die neu zusammengesetzten Arbeitschritte erlernen musste, sondern die Bewegung in Einzelbereiche untergliedert blieb, die mit Hilfe der Stoppuhr kontrolliert und optimiert wurden. ${ }^{56}$

\section{Die Bedeutung von Stoppuhr und Zeitstudie in der deutschen Rationalisierungsbewegung}

Im Vorwort der 1929 vom Ausschuß für wirtschaftliche Fertigung (AWF) herausgegebenen Veröffentlichung zum Thema „Zeitstudien“ wird betont: „Die Bedeutung der Zeitstudie für wirtschaftliche Betriebsführung wird in steigendem Maße von den verschiedenen Wirtschaftszweigen anerkannt. Sie ist Ausgangspunkt für die Entwicklung einwandfreier Unterlagen zur Durchführung einer von den Schwankungen wirtschaftlicher Verhältnisse vollkommen unabhängigen Betriebskostenvor- und -nachrechnung. Die Zeitstudie ermöglicht es, die richtige Zeit als unveränderlichen Maßstab für jede Art von Arbeit zu erfassen, und bildet so die Grundlage für die gerechte Entlohnung der geleisteten Arbeit. " ${ }^{57}$

Die in den Anfängen der Rationalisierungsbewegung gegründeten Institutionen erweiterten sich in dem Maße, in welchem sich die Zeitstudie als Rationalisierungsmittel ausdifferenzierte. Hier sei genannt der Ausschuss für Zeitstudien, der 1920 im AWF gegründet wurde, wobei sich die Forschungsgebiete bald in Hand- und Maschinenarbeit auftrennten. ${ }^{58}$ Parallel hierzu wurde 1924 vom Gesamtverband deutscher Metallindustrieller der Reichsausschuss für Arbeitszeitermittlung (REFA) gegründet. Dieser setzte sich zum Ziel, Unterlagen und Lehrmaterial bereitzustellen und die Ausbildung von Stückzeitrechnern und Zeitnehmern zu gewährleisten. Vier Jahre nach der Gründung des REFA wurde eine erste umfangreiche Veröffentlichung herausgegeben, die verbindliche Richtlinien für die Verwendung einer eindeutigen Fachterminologie, für die Auswahl eines befähigten Zeitnehmers, für die Wahl der geeigneten Hilfsmittel und für die Durchführung und Auswertung einer Zeitstudie gab. ${ }^{59}$ Ein Jahr später folgte eine Veröffentlichung des AWF, der die Richtlinien für eine Zeitstudie weitergehend für das gesamte Betriebsgeschehen aufschlüsselte und dem Aspekt der Rationalisierung mehr Platz einräumte. ${ }^{60}$ Die nun institutionell und hierarchisch festgelegte Zeiter-

56 Siehe hierzu Jürgen Bönig, Die Einführung der Fließbandarbeit in Deutschland bis 1933. Zur Geschichte einer Sozialinnovation, Bd. 1, Münster u. Hamburg 1993.

57 Ausschuß für Handarbeit (AWF) (Hg.), Grundlagen für Arbeitsvorbereitung, Zeitstudien, Berlin 1929, S. V.

58 Siehe hierzu Eduard Michel, Wie macht man Zeitstudien. Arbeits- und Zeitstudien zur genauen Festsetzung von richtigen Stücklöhnen in Maschinenfabriken, Berlin 1920. Eduard Michel war Berliner Ingenieur und Obmann des Ausschusses für Zeitstudien beim AWF.

59 Reichsausschuss für Arbeitszeitermittlung (Hg.), REFA-Buch, Einführung in die Arbeitszeitermittlung, Berlin 1928, S. 6. (Folgend als erstes REFA-Buch bezeichnet); Engelbert Pechhold, 50 Jahre REFA, Darmstadt 1974, S. 48-55.

60 AWF, Zeitstudien (wie Anm. 57). 
fassung sollte die Berechnung der Stückzeitbestimmung und der Akkordlöhne gleichschalten, Zeitnormen für elementare Arbeiten festlegen und die Arbeitsverteilung und die Zeitkontrolle aller Arbeitsabläufe in Büro und Werkstatt berechenbar und überprüfbar machen. In Abbildung 6 sind diese vier Bereiche aufgeschlüsselt dargestellt.

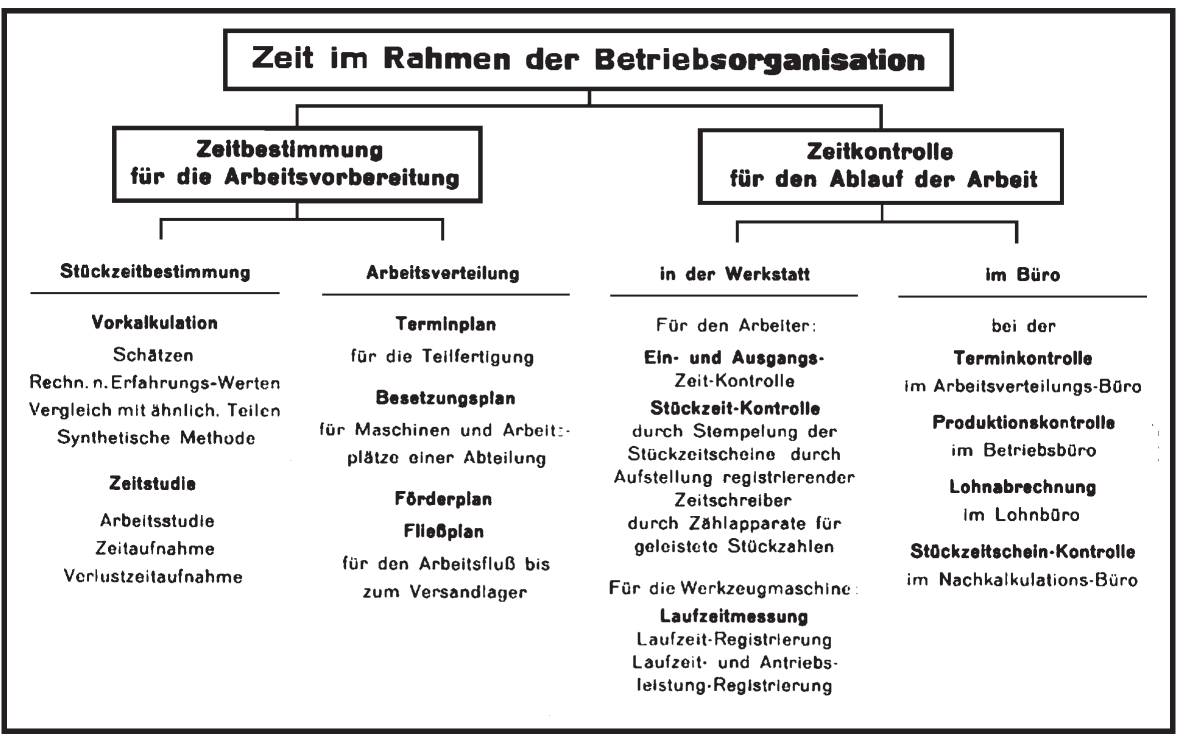

Abb. 6: Zeitliche Vernetzung im Betrieb. Quelle: Ausschuß für Handarbeit (AWF) (Hg.), Grundlagen für Arbeitsvorbereitung, Zeitstudien, Berlin 1929, S. 4.

Diese straffe, nach den Regeln der Zeit durchorganisierte Betriebsstruktur ist das tatsächliche, innovative und zugleich reglementierende und deterministische Bild der Rationalisierungsbewegung. Die Zeitstudie, die Taylor eingeführt hatte, wurde hiermit bis in die kleinsten Details weiter entwickelt und zum Knotenpunkt aller betrieblichen Veränderungen. Die Stoppuhr, die sich schon in der Debatte um Taylors Schriften als Zeichen für Arbeitszeitveränderungen herausgeschält hatte, wurde hier eindeutig zur Metapher für die zeitliche Determinierung des menschlichen Arbeitsprozesses.

\section{Die Entwicklung der Industrie-Stoppuhr}

Schon bei Taylor wurde auf die Zweckmäßigkeit einer Dezimalteilung des Ziffernblattes hingewiesen. ${ }^{61}$ Diese Einteilung blieb bei allen Stoppuhrmodellen für Arbeits- und Zeitstudien bestehen. Noch 1919 existierte aber in Deutschland keine Uhrenfirma, die Stoppuhren anbieten konnte, die den An-

61 Taylor, Betriebsleitung, (wie Anm. 39), S. 15, §44 und Kap. XII, Vornahme der Zeitstudien, S. 91-101, bes. S. 94f., §198. 
forderungen der Rationalisierungsinstitutionen entsprach..$^{62}$ Basierend auf den Grundanforderungen der Industrie stellte der AWF erste Richtlinien auf, die eine Industrie-Stoppuhr erfüllen sollte. Diese beinhalteten Größe, Zifferblatteinteilung und Farbe, Qualität und ergonomische Handhabung. ${ }^{63}$ Bis 1929 hatte der AWF gemeinsam mit der Uhrenindustrie verschiedene Stoppuhrmodelle für den Zeitnehmer entwickelt und sie mit den Bezeichnungen „AWF 0“ bis „AWF 4“ in den Vertrieb gebracht. ${ }^{64}$ Um die Unterschiede einiger Stoppuhrmodelle von 1920 und 1929 zu verdeutlichen, werden die Abbildungen der AWF-Stoppuhren (Abb.7b, 7c) mit einer älteren Stoppuhr in Vergleich gesetzt (Abb. 7a).

Unabhängig davon, wie eine Stoppuhr technisch ausgeführt ist, lassen sich auf ihr mehrere verschiedene Zeiten ablesen. Die Folge- oder Fortschrittszeiten werden mit einem kurzen Blick auf den laufenden Zeiger abgelesen. Einzelzeiten erhält man durch das Stoppen des Zeigers und die Rückstellung auf Null. ${ }^{65}$ Die nachfolgenden Stoppuhrmodelle veranschaulichen exemplarisch einen Teil der Ablesemöglichkeiten bei Stoppuhren im Arbeitsprozess:

AWF 0 (Kronenstopper, Abb. 7b) ist die einfachste Form einer Industriestoppuhr. Starten, Stoppen und Rücksetzen des Zeigers auf die Nullposition geschehen durch den Druck auf die Krone. Die Zeiten werden hier meist bei fortlaufendem Zeiger abgelesen. Oftmals werden zwei oder drei dieser Uhren gleichzeitig verwendet, um Einzelzeiten zu ermitteln und auf der dritten Uhr die Fortschrittszeit abzulesen.

AWF 2 (Doppelstopper oder Schleppzeigeruhr, Abb. 7c) besitzt einen zweiten Sekundenzeiger. Beide Zeiger können parallel laufen, aber zu beliebigem Zeitpunkt getrennt werden und auch getrennt auf Null zurückgesetzt werden, während der zweite Zeiger weiterläuft. Diese Uhr dient dem Messen sehr kurzer aufeinander folgender Zeiten, wie sie meist in der Massenfertigung vorkommen.

Andere Modelle - wie etwa AWF 1 (Additionsstopper) - sind mit einer Temposchaltung ausgestattet, bei welcher der Zeiger aus dem Lauf heraus

62 Michel (wie Anm. 58), S. 25f.; Drescher u. Boek, Stoppuhren zur Messung von Arbeitszeiten, in: Deutsche Uhrmacher-Zeitung 66, 1922, Nr. 9, S. 106ff. Thematisiert ebenfalls in Uhrmacherkreisen: o.A., Die Stoppuhr dem Uhrmacher, in: Deutsche Uhrmacher-Zeitung 68,1924 , Nr. 20, S. 289-291.

63 In Drescher/Boek (wie Anm. 52), S. 106 findet sich eine Auflistung; weitere Anforderungen sind zu finden in Die Stoppuhr dem Uhrmacher (wie Anm. 62), S. 291.

64 Die Datierung von 1929 führe ich auf die Veröffentlichung des AWF zurück, in der diese Bezeichnungen das erste Mal auftauchen, siehe AWF, Zeitstudien (wie Anm. 57), S. 30-35. Im ersten REFA-Buch von 1928 sind diese Bezeichnungen noch nicht mit aufgenommen worden. Siehe auch Ausschuß für wirtschaftliche Fertigung e.V., Richtlinien für die Ausgestaltung von Industrie-Stoppuhren (AWF 68), Berlin u. Frankfurt a.M. o.J. (Folgend als AWF 68 bezeichnet); Reichskuratorium für Wirtschaftlichkeit (RKW) (Hg.), Handbuch der Rationalisierung, Berlin 1930, S. 274, hier heißt es, dass der Zeitnehmer die Stoppuhren verwenden sollte, die vom AWF gemeinsam mit der Uhrenindustrie entwickelt wurden.

65 Siehe hierzu die ausführlichen Erläuterung in: AWF, Zeitstudien (wie Anm. 57), S. 100f. 


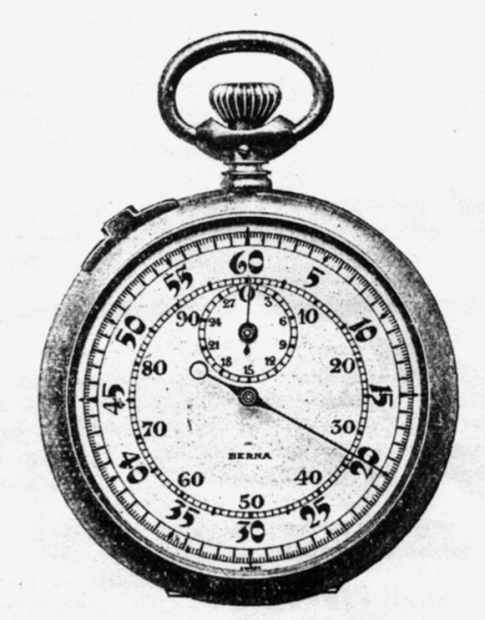

Abb. $7 \mathrm{a}$
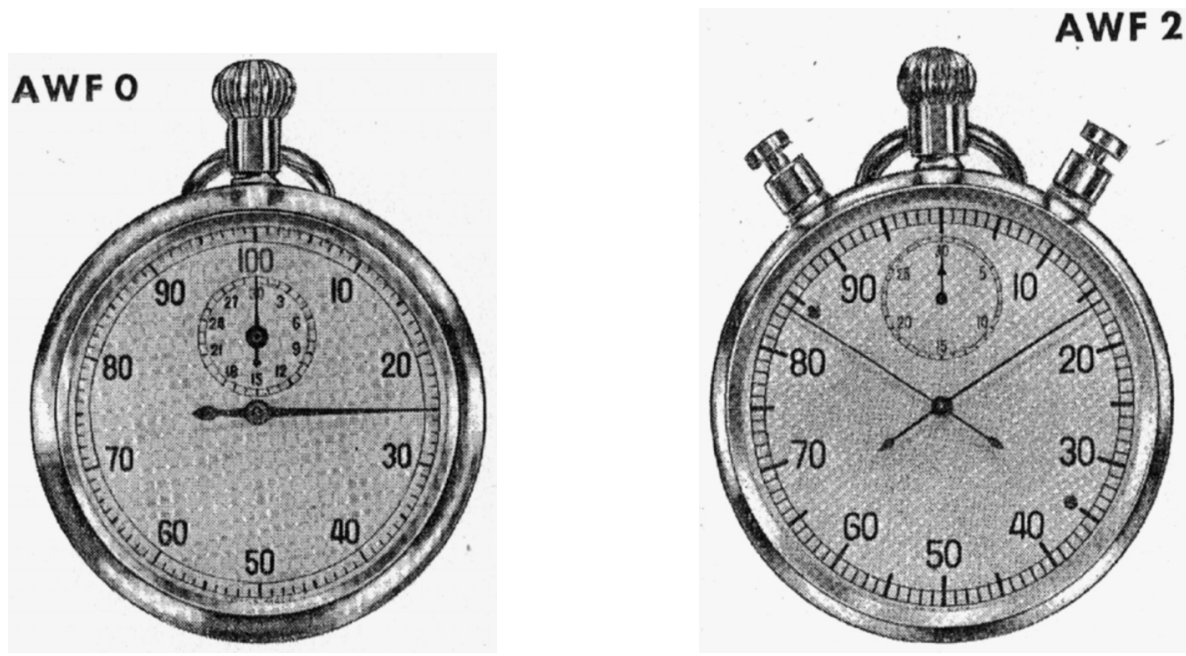

Abb. $7 b$

Abb. 7c

Abb. 7: Stoppuhren für die Zeitstudie. Quelle: 7a aus: o.A., Stoppuhren für Zeitstudien, in: Die Uhrmacherwoche, 1922, Nr. 28, S. 467; 7b, 7c aus: Ausschuß für wirtschaftliche Fertigung e.V. (Hg.), Richtlinien für die Ausgestaltung von Industrie-Stoppuhren (AWF 68), Berlin u. Frankfurt a.M. o. J., S. 4 u. 5.

auf die Nullstellung gebracht werden kann, und dann ohne Verzögerung von dort aus weiterläuft. Diese Uhr ist nach den Richtlinien des AWF für Beobachtungen in der Einzel-, Reihen- und Massenfertigung geeignet. ${ }^{66}$

66 AWF 68 (wie Anm. 64), S. 4ff.; AWF, Zeitstudien (wie Anm. 57), S. 31 ff.; Heinz (wie Anm. 5), S. 13-16. 
Zusätzlich zu den unterschiedlichsten Stoppuhren wurden viele unterschiedliche Zeitmesser zur Rationalisierung und Kontrolle der Arbeitsprozesse entwickelt. In Abbildung 8 werden alle gängigen Zeiterfassungsgeräte, die zur Rationalisierung und Prozessoptimierung 1929 einsetzbar waren, klassifiziert.

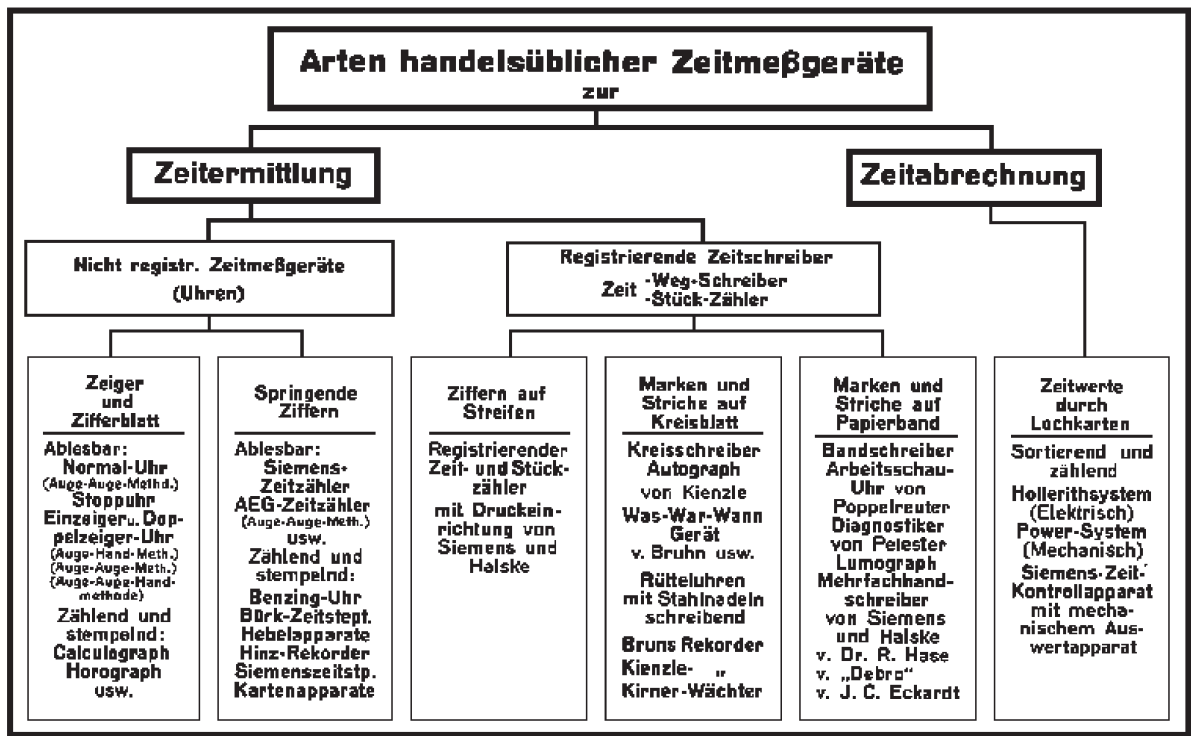

Abb. 8: Handelsübliche Zeitmessinstrumente geordnet nach Konstruktionsmerkmalen, 1929. Quelle: Ausschuß für Handarbeit (AWF) (Hg.), Grundlagen für Arbeitsvorbereitung, Zeitstudien, Berlin 1929, S. 29.

Die Zeitaufnahme mit Hilfe der Stoppuhr erfolgte immer durch einen Zeitnehmer (REFA-Mann), der unmittelbar in nächster Nähe des Arbeitenden stand. Da der REFA-Mann im Betrieb eine hohe Vertrauensposition einnehmen sollte, wurden genaue Kriterien zur Auswahl eines Zeitnehmers getroffen, von denen nur eine geringe Zahl wiedergegeben werden kann. Geistige Fähigkeiten, wie etwa eine klare schriftliche und mündliche Ausdrucksweise, oder ein praktisch-kritischer Blick wurden ebenso geprüft wie charakterliche Anforderungen. Hierzu gehörten unter anderem: Takt, eine positive Einstellung, Gerechtigkeitsempfinden und ein zielbewusstes Wesen, das nicht in Eigensinn ausarten sollte. ${ }^{67}$ Arbeit, Uhr, Beobachtungsbogen und Auge des Beobachters sollten durch eine imaginäre gerade Linie verbunden sein. Dem Zeitnehmer standen verschiedene Verfahren zur Verfügung, denen bestimmte Stoppuhren zugeordnet waren (siehe auch Abb. 7). Hier wäre das „Auge-Hand“Verfahren, bei welchem die Stoppuhr beim Beobachten eines Zeitabschnittes angehalten und Einzelzeiten abgelesen wurden, oder das „Auge-Auge“-Verfahren, bei dem mit Hilfe der Stoppuhren AWF 1 oder AWF 2 Fortschritts-

67 AWF, Zeitstudien (wie Anm. 57), S. 24f. 
zeiten abgelesen wurden, zu nennen. Diese verschiedenen Methoden wurden unter den Aspekten einer Reaktionszeitmessung im Laboratorium für Industrielle Psychotechnik der Technischen Hochschule Berlins untersucht, um ihre Messgenauigkeiten zu vergleichen. ${ }^{68}$

\section{Die Durchführung der Zeitstudien}

Wie wurde nun auf der Basis der geschaffenen Grundlagen die Zeitstudie mit der Stoppuhr durchgeführt? ${ }^{69}$ Welche Arbeitsprozesse wurden gemessen? Die Zeitstudie beschäftigte sich nicht ausschließlich mit der zeitlichen Erfassung und Verkürzung einzelner Griffelemente, sondern ihr erstes Anliegen war die Aufnahme des Arbeitsprozesses in der ganzen Linie. ${ }^{70} \mathrm{Nach}-$ folgend sind die vier verschiedenen Stufen einer Zeitstudie aufgeführt, wobei die größte Aufmerksamkeit dem ersten Punkt, der Arbeitsstudie (Arbeits-

68 o.A., Die Psychotechnik des Zeitnehmens in der Werkstatt, in: Deutsche Uhrmacherzeitung 36, 1928, S. 689f.; M. Moisecu, Psychotechnik des Zeitnehmens in der Werkstatt: Vergleich verschiedener Zeitmeßinstrumente und Zeitmeßverfahren, in: Industrielle Psychotechnik 4, 1927, H. 4, S. 97-121. Das Ohr-Auge-(Hand-)Verfahren wurde bei astronomischen Beobachtungen verwendet. Der Beobachter zählte akustische Sekundenschläge (Ohr) während der Beobachtung (Auge) und stoppt die Uhr bei Beendigung der Beobachtung (Hand). Zur Verflechtung staatlicher und wirtschaftlicher Institutionen siehe Rainer W. Hoffmann, Wissenschaft und Arbeitskraft. Zur Geschichte der Arbeitsforschung in Deutschland, Frankfurt a.M. 1985, bes. Kap. 5.

69 Aufbauend auf den Taylorschen Zeitstudien entwickelte der in den USA lebende Franzose Charles Bedaux (1886-1944) um 1916 ein umfangreiches Zeitstudiensystem, bei dem die Leistung nicht nach dem Zeitaufwand für ein Stück ermittelt wurde, sondern nach einer eigens entwickelten Leistungseinheit (nach ihrem Erfinder kurz „B“ genannt), die sich aus drei Faktoren (Arbeit, Erholung, Geschwindigkeit) zusammensetzte. Auch hier stand am Anfang eine Zeitstudie, welche die Arbeit in Teilbereiche aufschlüsselte und der Rationalisierung diente. Im Unterschied zur REFA-Studie war beim Bedaux-System der Rechenweg nicht nachvollziehbar und nicht zugänglich. Gerade der Erholungskoeffizient wurde von der Bedaux-Gesellschaft streng geheim gehalten, er war das Firmenkapital. Aufgrund der überaus schwierigen und schwer nachvollziehbaren Kalkulationsgrundlagen fand das Bedaux-System in der deutschen Industrie wenig Anklang. (Zum System und einzelnen Aspekten vgl. Arbeitswissenschaftliches Institut der DAF (Hg.), Das Bedaux-System. Eine gutachtliche Stellungnahme, Berlin 1937; Paul Erker, Das Bedaux-System. Neue Aspekte der historischen Rationalisierungsforschung, in: Zeitschrift für Unternehmensgeschichte 41, 1996, S. 139-158; Erwin Rochau, Das Bedaux-System, Praktische Anwendung und kritischer Vergleich mit dem REFA-System, Würzburg 1952; Helmut Kunze, Bedaux-Verfahren. Der neue Weg. Steigerung der Produktivität, Haar bei München 1977).

70 Die Begrifflichkeiten zur Zeitstudie, insbesondere in der frühen Phase, variieren mit fortlaufender Weiterentwicklung. Siehe hierzu AWF, Zeitstudien (wie Anm. 57), S. 72ff. Hier wird eindeutig festgehalten, dass die Zeitstudie aus zwei Elementen besteht, der Arbeitsstudie und der Zeitaufnahme. - Zur REFA-Zeitstudie vgl. auch Pechhold (wie Anm. 49), S. 71-74; vgl. ebenfalls erstes REFA-Buch (wie Anm. 59), S. 25. Hier soll vor der Zeitaufnahme eine Arbeitsaufnahme mit Hilfe einer Bewegungsstudie durchgeführt werden. Im zweiten REFA-Buch von 1939 (Reichsausschuß für Arbeitszeitermittlung (Hg.), Zweites REFA-Buch. Erweiterte Einführung in die Arbeitszeitermittlung, Berlin 1939) wird dagegen der Begriff Zeitstudie nicht mehr ordnend einem Kapitel überschrieben. Die Zeitstudie wird hier allgemeiner, als eine systematische Zeitbeobachtung bezeichnet. 
aufnahme) und dem dritten Punkt, der Zeitaufnahme gilt: In der Arbeitsaufnahme oder Arbeitsstudie wurde der gesamte Arbeitsprozess durch den Zeitnehmer analysiert. Dies beinhaltete alle Komponenten, die die Leistung des Arbeiters beeinflussten, zum Beispiel sanitäre Anlagen, Werkzeuge und Maschinen, aber auch Licht- und Wärmeverhältnisse spielten eine Rolle. Das Ergebnis, eine erste Unterteilung der Gesamtfertigung in einzelne Arbeitsschritte, wurde in eine Arbeitsunterweisungskarte eingetragen, die der Arbeiter gemeinsam mit dem Arbeitsauftrag erhielt. ${ }^{71}$

Der zweite Schritt war die Rationalisierung des beobachteten Arbeitsablaufes und die Kontrolle anhand der Arbeitsunterweisungskarte, die der Arbeiter bei Annahme des Arbeitsauftrages erhielt.

Den dritten und wichtigsten Schritt stellte die Zeitaufnahme der rationalisierten Arbeitsabläufe und die Eintragung der Zeitwerte in den Beobachtungsbogen dar. Die Zeitaufnahme war (vgl. Abb. 9) in unterschiedliche Abschnitte unterteilt. Die Grundzeit bildete das wichtigste Element der Stückzeit, da sie die genaue Fertigungszeit für einen Arbeitsgang bezeichnete. Im Gegensatz dazu stand die Nebenzeit, welche regelmäßig auftrat, aber bei der keine Arbeitsmerkmale im Sinn des Fertigungsauftrages entstanden, z.B. Einführen eines Bleches per Hand in eine Stanze. Zusätzlich zu allen den Arbeitsablauf fördernden Zeiten musste der Zeitnehmer eine Verlustzeit ermitteln, die sich unregelmäßig und betriebsbedingt ergab. ${ }^{72} \mathrm{Zu}$ Verlustzeiten wurde die Wartung von Werkzeug und Maschine gerechnet sowie persönliche Pausen, hierzu zählten auch Gespräche mit dem Vorgesetzten. Sie wurden aufgegliedert in ,abzugeltende', , von Fall zu Fall abzugeltende' und ,nicht abzugeltende" Verlustzeiten. Zu letzteren gehörten nach einer Liste des REFA beispielsweise unnötige Gespräche mit Kollegen und das Waschen außerhalb der Pausenzeiten. ${ }^{73}$ Intensiver noch als bei einer Zeitaufnahme der Arbeit wurde der Arbeiter vom Zeitnehmer mit der Stoppuhr verfolgt, und es wurden alle individuellen, nicht unmittelbar arbeitsrelevanten Zeiten, wie etwa Gespräche mit Kollegen, aus der Verlustzeitrechnung gestrichen. Die Verlustzeitaufnahme war in erster Linie ein Mittel des Arbeitgebers, den Arbeiter davon abzuhalten, bewusste Verzögerungen, wie den Plausch mit dem Lagerverwalter beim Abholen eines Werkzeuges, zu vermeiden, da er zusätzliche Produktionspausen nicht entlohnen wollte. Umgekehrt konnte auch der Arbeiter eine Verlustzeitaufnahme beantragen, wenn ihm etwa das zu lange Anstehen an der Stempeluhr als nicht abzugeltende Verlustzeit verlorenging.

71 Die Anforderungen und Komponenten der Arbeitsstudie wurden ausgewählt aus AWF, Zeitstudien (wie Anm. 57), S. 72-89.

72 Eine genaue Aufschlüsselung der Fertigungszeit findet sich ebd., S. 12-20.

73 Erläuterung und Auflistung der verschiedenen Verlustzeiten sind im zweiten Refa-Buch (wie Anm. 70), S. 24-27 enthalten; siehe auch Kurt Hegner, Lehrbuch der Vorkalkulation von Bearbeitungszeiten, Bd. 1: Systematische Einführung, (Schriften der Arbeitsgemeinschaft Deutscher Betriebsingenieure, Bd. 2), Berlin 1924, S. $13 \mathrm{f}$. 


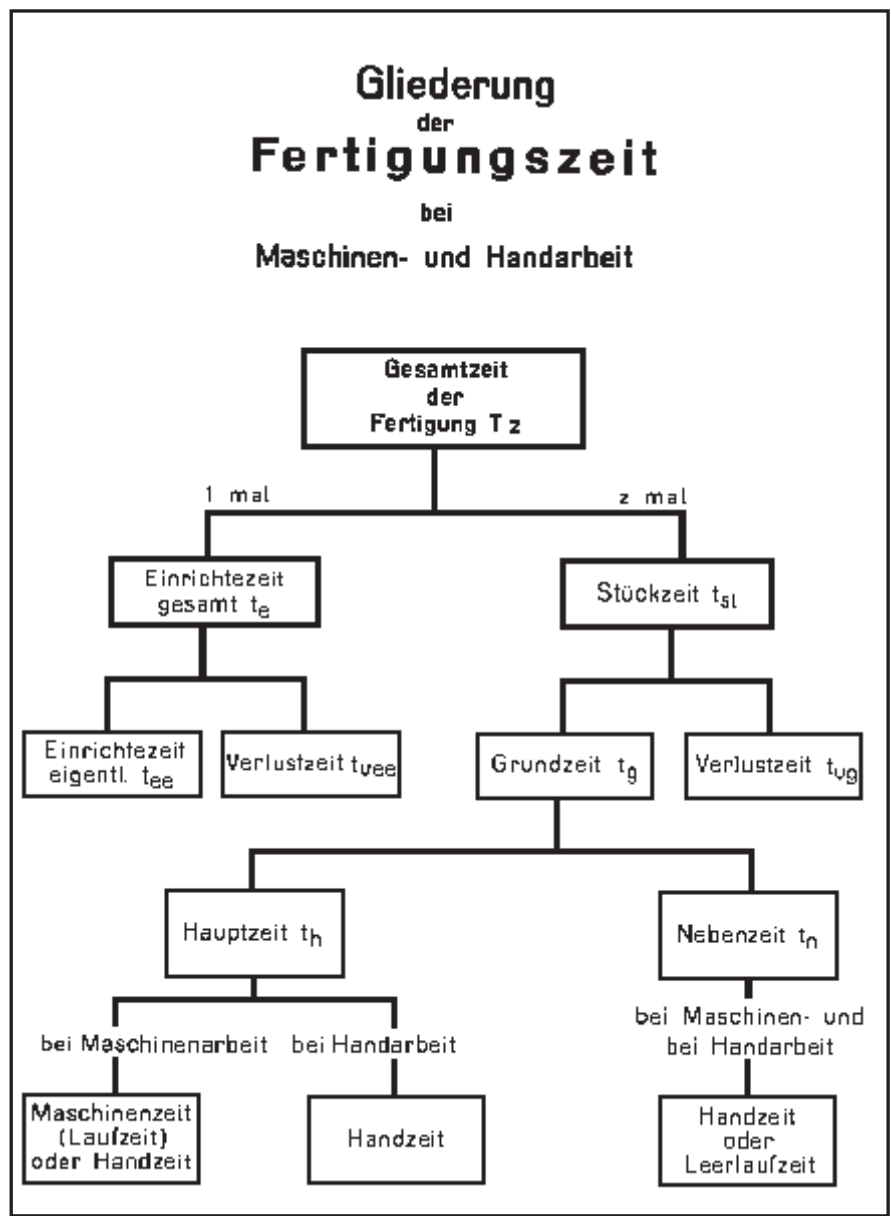

Abb. 9: Unterteilung des Arbeitsprozesses. Quelle: Ausschuß für Handarbeit (AWF) (Hg.), Grundlagen für Arbeitsvorbereitung, Zeitstudien, Berlin 1929, S. 14.

Diese Überprüfung hätte bei gerechtfertigter Beschwerde einen Zeitzuschlag bewirkt. Gerade Zeitzuschläge sind auch noch heute einer der schwierigsten Teilbereiche der Akkordvorgabe und sie bilden den größten Streitpunkt zwischen Arbeitnehmer und Arbeitgeber, da jeder Materialfehler, jede Maschine mit Mängeln, jede Wartezeit und jede unregelmäßige Unterbrechung der Arbeit als unterschiedliche Verlustzeit ausgelegt und angerechnet werden können. ${ }^{74}$

74 Ebd.; Harold B. Maynard (Hg.), Handbuch des industrial engeneering, Berlin 1956, S. 55-59. Hier werden Zeitzuschläge für Verlustzeiten nun als Verteilzuschläge bezeichnet und nach wie vor mit einer Beispielliste veranschaulicht. Hier heißt es weiterhin: „Der Zeitstudieningenieur sollte sorgsam vermeiden, dass die Verteilzeiten zu einem bequemen Tummelplatz für all die Fehler und Nachlässigkeiten werden, die bei einer Analyse einer Arbeit aufgetreten sind.“ 
Bevor der Zeitnehmer mittels der oben erläuterten Verfahren und Hilfsmittel die verschiedenen Griffzeiten in der Werkstatt stoppte, trug er die in der Arbeitsstudie ermittelten Griffelemente in den Beobachtungsbogen ein. Die Spaltenzahl für die Häufigkeit des zu beobachtenden Arbeitsgangs richtete sich nach der Produktionsform. So wurden für die Einzelfertigung nur fünf Spalten, also fünf gleiche Beobachtungsvorgänge, benötigt. Für die Zeitaufnahme in der Reihenfertigung benötigte man zehn und in der Massenfertigung nicht unter zwanzig Zeitaufnahmen des gleichen Arbeitsablaufs. Die Einrichtzeit sowie alle Zeiten zum Vorbereiten der Arbeit an der Maschine wurden auf der Vorderseite eingetragen und im günstigsten Fall nur einmal gemessen. ${ }^{75}$

Die vierte Stufe der Zeitstudie war schließlich die Auswertung der gemessenen Zeiten, der Aufbau eines Tabellenwerkes für Richtzeiten, die Nachprüfung der Gebrauchstabellen vor Herausgabe an das Kalkulationsbüro durch Kontrollmessungen. ${ }^{76}$

\section{Schluss}

„Da auch nicht die kleinste Handreichung mehr als selbstverständlich hingenommen, sondern zum Gegenstand genauer Untersuchung und Zeitmessung gemacht werden muß, so ist die Stoppuhr, die jede Bewegung in Bruchteilen von Sekunden mißt, zum Wahrzeichen, zum Grundstein dieser neuen technischen Wissenschaft geworden. Ebenso wie die Bearbeitungsmaschinen allmählich so umgestaltet worden sind, daß die größtmögliche Ausnutzung der natürlichen Bewegungszuordnung erreicht werden konnte, muß auch der Muskelmechanismus des Menschen auf den günstigsten Wirkungsgrad einstellbar sein. ${ }^{677}$ Damit hatte Schlesinger 1913 das Potential der Stoppuhr für die wissenschaftliche Betriebsführung und die Analyse von Arbeitsprozessen präzise formuliert. Im Zuge der Rationalisierungsbewegung wurden diese von Schlesinger formulierten Ansätze für die Bearbeitungsmaschinen und die körperliche Arbeit weiter entwickelt und am Arbeiter „eingestellt“. Die Impulse und Grundlagen für den Einsatz der Stoppuhr im Arbeitsprozess kamen dabei aus unterschiedlichen Bereichen: aus dem sportlichen Bereich trugen Leistungsmessung und Leistungsoptimierung dazu bei, im militärischen Bereich forcierten Uniformierung bzw. Gleichförmigkeit und Synchronisation von Abläufen sowie Disziplinierung die präzise Zeitmessung und schließlich erlangte die Optimierung der Zeitanzeiger im wissenschaftlichen Kontext zunehmend an Bedeutung.

75 AWF, Zeitstudien (wie Anm. 57), S. 95-99 u. 109-120; Zweites REFA-Buch (wie Anm. 70), S. 61-72; Hegner (wie Anm. 73), S. 87-93.

76 Zitate aus erstes REFA-Buch (wie Anm. 59), S. 25.

77 Georg Schlesinger, Betriebsführung und Betriebswissenschaft, in: Technik und Wirtschaft. Monatsschrift des VDI 6, 1913, H. 8, S. 534. 
Das Potential der Stoppuhr zur Rationalisierung industrieller Arbeitsprozesse, das Schlesinger 1913 konstatierte, wurde im Zuge der Rationalisierungsbewegung entfaltet, und die unscheinbare „Stoppuhr“, die bisher in der Forschung kaum gewürdigt wurde, galt spätestens mit der Kollage von John Heartfield „Ein Gespenst geht um in Europa“ als die Metapher für die Rationalisierung schlechthin.

Anschrift der Verfasserin: Sonja Apel, Cheruskerstraße 20, 10829 Berlin, EMail: apel.sonja@gmx.de 
